Lena Klopp-Schulze, Charlotte Kloft

Abt. Klinische Pharmazie und Biochemie, Institut für Pharmazie, Freie Universität Berlin, Berlin, Deutschland

\title{
Erratum zu: CYP2D6-Genotypisierung vor Tamoxifen-Therapiestart
}

\section{Erratum zu:}

best practice onkologie 2018

https://doi.org/10.1007/s11654-018-0102-x

Durch einen technischen Fehler wurde die Autorenschaft in der XML-Version des Beitrags nicht korrekt dargestellt. Wir bitten Sie die korrigierte Autorenschaft zu beachten und den Fehler zu entschuldigen.

\section{Korrespondenzadresse}

\section{Prof. Dr. Charlotte Kloft}

Abt. Klinische Pharmazie und Biochemie, Institut für Pharmazie,

Freie Universität Berlin

Kelchstraße 31, 12169 Berlin, Deutschland

charlotte.kloft@fu-berlin.de 